\title{
BCG: The Optimal Approach in Superficial Bladder Cancer
}

\author{
A. Böhle \\ Chairman
}

Floor: Dr Rischmann recommends urine culture if fever persists following BCG installation. Would not blood culture for mycobacteria also be appropriate if you are concerned about systemic BCG septicaemia?

P. Rischmann: If fever and side-effects such as cystitis are moderate, then you should proceed with urine culture. While waiting for the results, fluoroquinolones and paracetamol can be given. In the case of severe systemic effects, one should not wait for bacteriological results before beginning treatment. Blood cultures are seldom positive; reverse-transcriptase-PCR may be more useful.

A. Böhle: From our own experience, and from the experience in the literature, blood cultures are not very successful at revealing mycobacterial infection. Blood cultures are often negative even in the presence of systemic BCG infection.

Floor: Is any of the various BCG strains superior? Furthermore, when referring to reductions in the dose of $\mathrm{BCG}$, does it make more sense to talk about colony-forming units (CFUs) rather than milligrams? Equal doses (in milligrams) of different strains of BCG may have different numbers of CFUs.

P.F. Bassi: Which parameters we use to measure BCG is an important topic, but one that is still largely unexplored. As a matter of fact, we do not know what the active component of the BCG is or how BCG works. As a consequence, we do not know what to measure: weight is only a manufacturer's product information, which does not express the absolute therapeutic ability of the prepara- tion. It is, therefore, not possible to make a comparison by weight between, for example, $150 \mathrm{mg}$ of Pasteur BCG, 81 $\mathrm{mg}$ of Connaught BCG or $50 \mathrm{mg}$ of OncoTice. Consequently, it is impossible to say that $150 \mathrm{mg}$ Pasteur BCG is threefold 'better' than $50 \mathrm{mg}$ Tice strain. Also the number of viable colonies (CFUs) has been supposed, in the past, to make a difference in terms of efficacy, but in the long run, this highly imprecise parameter has failed to be a reliable indicator.

D.L. Lamm: The variability not only between strain and strain, but also between one ampoule and another ampoule of the same preparation is very high. For this reason, Mike O'Donnell's recommendation for dose reduction makes a lot of sense, and in our experience is very useful. When patients start to have increased side-effects we reduce the dose to one third; if side-effects persist or increase, the dose is reduced to one tenth, and even to one hundredth if necessary. Basically, the patient does not have to suffer at all to receive effective immune stimulation with BCG, and I think that dose reduction therefore really makes more sense than prophylactic isoniazid. With isoniazid we are adding another variable, and we don't know what percentage kill isoniazid prophylaxis results in, unlike log reductions in BCG dose, which do give us a rough idea of the effective decrease in dose.

Floor: Do you think there is any correlation between the pre-treatment tuberculin test and the subsequent development of side-effects?

\begin{tabular}{ll}
\hline KARGER & ( ) 2000 S. Karger AG, Basel \\
Fax +4161306 1234 & \\
$\begin{array}{l}\text { E-Mail karger@karger.ch } \\
\text { www.karger.com }\end{array}$ & $\begin{array}{l}\text { Accessible online at: } \\
\text { www.karger.com/journals/eur }\end{array}$
\end{tabular}


D.L. Lamm: There is some correlation between PPD response and side-effects as well as immune response and anti-tumour effect, though this is not a one-to-one correlation. Generally speaking, you need to have some irritative symptoms to be certain that you are stimulating an immune response. Those patients who have been previously exposed to $\mathrm{BCG}$ will typically have a more rapid and more vigorous response to BCG. In patients with carcinoma in situ (CIS), for example, or patients with highrisk bladder cancer, those that were PPD-positive have a better response than those who were negative, and certainly there is a correlation between response and conversion of the skin test to positive after BCG. Experience from the Southwest Oncology Group (SWOG) shows that patients with CIS who converted to a positive PPD skin test had a $30 \%$ higher response than those who failed to convert - so the PPD is of some use.

Floor: What percentage of patients in the SWOG study had muscle invasive disease on subsequent biopsies during maintenance BCG? Did the drop-outs in this study occur at a particular time after initial presentation? Also, what percentage of patients completed the three-year regimen?

D.L. Lamm: The drop-out rate was $84 \%$, and that was partly by intention. Investigators were specifically instructed not to give the second or the third instillation if there were increased side-effects from the first. Therefore, only $16 \%$ received BCG at the 36th month. We don't have the specific percentages that had cystectomy vs. documented muscle invasion vs. requirement of systemic chemotherapy for clinical metastatic disease. The majority had muscle invasion, though a significant number did have implied progression based on those other factors.

Floor: Of those who proceeded to cystectomy, what percentage did not have muscle invasive disease, but kept experiencing recurrences despite being on the maintenance therapy?

D.L. Lamm: I don't have those exact data, but there are patients who proceed to cystectomy because of recurrent CIS, grade 3 TCC or lamina propria invasion.

Floor: Dr Rischmann has mentioned haematuria as a contraindication to BCG therapy. Is microscopic haematuria also a contraindication?

P. Rischmann: No, only macroscopic haematuria is a contraindication. BCG instillation must not be performed after traumatic catheterization. Dipstick analyses always show microscopic haematuria.

A. Böhle: However, you should of course treat and not wait in the case of CIS associated with microhaematuria. Basically, we are concerned that BCG does not enter the bloodstream, either after traumatic catheterization or through an open vessel caused by inflammation.

Floor: Is the addition of prednisolone recommended to treat patients with immunoallergic arthritis secondary to BCG? In the last few years, three of our patients have had very good responses to this corticosteroid.

P. Rischmann: We use prednisolone or corticosteroids in cases of sepsis or severe immunoallergic reaction. Moderate reactions such as arthralgia respond well, in our experience, to antihistamines and non-steroidal antiinflammatory drugs.

Floor: What is the recommended protocol for a patient who experiences recurrences at 1,2 and 3 years after an initial 6-week BCG course?

D.L. Lamm: Part of the rationale for three weekly instillations is the heightened immune response to the secondary exposure to BCG. For this reason, for patients who responded well to a single 6-week course but have subsequently failed or have not been on maintenance, I recommend, not a second 6-week course, but two 3-week courses separated by an interval of 3 months, followed by 3 -week maintenance at 6 months. However, the protocol does depend on how long it has been since the patient's initial exposure to BCG. If you look at the cytokine response, those that have had BCG more recently will respond earlier in the series of subsequent treatments. Therefore, if it has been many years since a patient had intravesical BCG, you may need to continue with 4 or 5 treatments until you start to have symptoms.

Floor: We heard that BCG is sensitive to fluoroquinolones, and I took that to imply that we should not use fluoroquinolones to treat a proven UTI, as this would reduce the BCG effectiveness; yet in France the recommendation is that a fluoroquinolone should be used to treat a proven UTI.

A. Böhle: The important point that we wished to convey is that fluoroquinolones interact with BCG. Whether you want to kill BCG depends on the severity of the concomitant inflammation or infection. Certainly there are situations where you do not want to kill BCG, and in such cases fluoroquinolones should not be administered. In contrast, epididymitis is very easily resolved with fluoroquinolone treatment. Testicular loss did occur in the earlier series of our patients; however, severe epididymitis or testicular loss did not occur after we started to use fluoroquinolones to treat initial testicular symptoms. So it makes sense to use fluoroquinolones if you want to kill BCG very early.

P. Rischmann: Severe side-effects occur most often after the third day. At that time, in my opinion, eradicat- 
ing BCG will not significantly reduce the efficacy of the immunological reaction which has already begun.

Floor: The French recommendations for side-effects management advise that granulomatous prostatitis should be treated with isoniazid and rifampicin for 3 months - but what do you mean by granulomatous prostatitis? If you do a rectal examination after BCG, you feel nodules in the majority of patients, and biopsies show changes characteristic of granulomatous prostatitis.

P. Rischmann: We agree that nearly every patient has histological granulomatous prostatitis. However, the French recommendations focus on patients with grade III adverse events, that present with fever or other systemic symptoms and a clinically apparent prostatitis. Such a severe, symptomatic adverse event occurs in about $1-3 \%$ of cases and requires treatment.

A. Böhle: At least $40 \%$ of patients receiving BCG will have asymptomatic granulomatous prostatitis, but no patient has ever been reported in the literature as having symptomatic granulomatous prostatitis as a sole event. No treatment is necessary for patients with asymptomatic granulomatous prostatitis alone. However, patients who present with severe symptoms should of course be treated.

Floor: In the future will it be possible to individualize BCG treatment for each patient?

A. Böhle: The EBIN group is working on this. Of course we need some prognostic indicators of when the BCG treatment course has been optimally completed, and we are not at that point yet. Examples are interleukin-2, cytokine titres and immune response in the bladder.
Floor: At what stage would you go over to cystectomy? Bill Catalona's group has previously reported that if a cystoscopic evaluation shows recurrence after an initial 6week induction course, then patients should receive another 6-week induction course; if they then again recur, you should go over to cystectomy. Is this still the feeling?

D.L. Lamm: It is true that a patient who has T1 or CIS at 6 months or beyond has a markedly increased risk for progression. I think it is also important to state that 'failure' at 3 months may not be failure. In our experience, and in the SWOG experience of patients with CIS at 3 months, the complete response rate will increase by $14 \%$ at 6 months even without additional BCG, and if we give three more BCG instillations the response rate will increase by an additional $30 \%$. So clearly 3 months is still too early. As a rule of thumb, 6 months would be correct, but in fact we have seen patients who have taken 9 months to effect a complete response. If a patient shows continual improvement - for example from symptomatic CIS where every biopsy is positive to biopsies where half are positive to one focal remaining positive biopsy at 6 months - I would not insist that the patient have a cystectomy.

Floor: Please could you provide some more information on the use of aspirin to prevent side-effects.

A. Böhle: We do not use aspirin prophylactically. We use it only as a mild analgesic, for example if a patient experiences pain owing to inflammation. Here, however, we have had very good experience with it, and so we recommend oral acetyl salicylic acid. 